\title{
Améliorations récentes apportées au logiciel NUNIEAU pour la numérisation des marégrammes papiers
}

\author{
Frédéric Pons
}

Centre d'Etudes Techniques de l'Équipement Méditerranée

Département Risques Eau Construction / Service Hydraulique

Pôle d'Activité CS 7049913593 Aix-en-Provence cedex 3

frederic.pons@developpement-durable.gouv.fr

\section{Résumé :}

Le logiciel NUNIEAU mis en ligne sur Internet et disponible en Français et en Anglais a été développé au CETE Méditerranée pour numériser des enregistrements papiers de niveaux marins dans le Golfe du Lion. Cet article fait le point sur les dernières avancées techniques disponibles dans NUNIEAU pour le traitement des données anciennes en s'appuyant sur des nettoyages semiautomatiques ou basés sur la marée prédite. Il propose une grille d'analyse du temps de traitement des marégrammes en fonction de leur genre. Le logiciel NUNIEAU peut aussi être utiliser pour des séries de hauteur d'eau en rivière ou pour des séries de température.

Les perspectives d'analyse de la marée pour la validation des données sont ensuite évoquées dans le cas particulier de données avec peu de suivi régulier et suivant l'état de l'art de la discipline.

\begin{abstract}
:
NUNIEAU software, available on CETE Mediterranee website in French and English was developed to scan and digitise tide gauge charts first in the Gulf of Lion. The last improvements available in NUNIEAU are described in this paper and are based on methods to suppress automatically or with tide prediction false information on old chart records. Spent time to digitise old chart records are estimated for each case of charts. NUNIEAU can also be used for river level or temperature series.

Tide treatment is also tested to validate old chart data digitise with few of recommendation for the following of sea level.
\end{abstract}

\section{Mots-clés :}

Niveaux marins - Numérisation - Marégrammes -Marée - Données historiques 


\section{$1 \quad$ Introduction}

L'enregistrement des niveaux d'eau en mer a débuté il y a plus de deux siècles pour diverses raisons (POUVREAU, 2008) comme l'analyse de la marée, les levés bathymétriques, la connaissance des conditions hydrodynamiques devant des ouvrages projetés... Ces dernières décennies, la problématique de risques et de remontée du niveau marin est venue s'ajouter aux raisons initiales. Les données de niveaux marins dont il est question dans le cadre de cet article ont été levées grâce des marégraphes mécaniques papiers qui enregistraient sur une feuille appelée marégramme le niveau marin par application d'un stylet sur un tambour tournant avec un système d'horloge. Ces données étaient traitées soit par lecture directe sur le marégramme, soit par des tables à digitaliser avec la récupération d'une valeur à pas de temps constant. Dans le cadre de ses interventions pour le compte de la Direction Régionale de l'Equipement (DRE) du Languedoc-Roussillon et par la suite du Service Central Hydrométéorologique d'Appui à la Prévision des Inondations (SCHAPI) et de la Direction Départementale de l'Equipement (DDE) des Bouches du Rhône, le CETE Méditerranée a mis au point le logiciel NUNIEAU (NUmérisation des NIveaux d'EAU) pour capitaliser plus rapidement l'ensemble des informations issues d'un marégramme dans l'optique des motivations anciennes et nouvelles de suivi du niveau marin (ULLMANN \& al, 2005 ; PONS, 2008).

Le logiciel NUNIEAU a été développé au CETE Méditerranée pour récupérer au départ des enregistrements papiers de niveaux d'eau dans le Golfe du Lion. Il a ensuite été adapté à la récupération des niveaux d'eau en rivière et à un ensemble (sans doute non exhaustif) de formats de marégramme ou limnigramme (feuilles inférieures au format A3, Noir et Blanc, feuilles mutli-signaux, rouleaux de plusieurs dizaines de mètres...).

Le CETE Méditerranée, fort de son expérience avec quelques 150 années récupérées a mis à disposition gratuitement sur son site Internet (http://www.cetemediterranee.fr/fr/rubrique.php3?id rubrique $=113$ ) cet outil en version française et anglaise (bêta version) qui a d'ores et déjà été téléchargé une soixantaine de fois par des organismes ou bureaux d'études français ou étrangers. L'objet de cet article est de rappeler le principe de l'outil, mais surtout de faire le point aujourd'hui sur ses évolutions et améliorations « industrielles » et les perspectives de validation de ces données.

\section{$2 \quad$ Principes du logiciel}

L'utilisation du logiciel NUNIEAU nécessite préalablement de scanner des marégrammes. Cette étape est un point important du processus qui sera vu ultérieurement. 
Ensuite le logiciel NUNIEAU adopte le principe de calage d'une image sous un logiciel SIG, à savoir qu'à l'image est associée un fichier de calage qui rassemble les informations nécessaires et vérifiables. Ce calage est basé sur une fiche de renseignement (date de pose, échelle, commentaires divers...) et la récupération d'une bande de couleurs du niveau marin tracé sur la feuille. A ceci s'ajoutent des outils de nettoyage nécessaires pour traiter des données anciennes (saisie par polygone, rectangle...)

La numérisation issue de NUNIEAU se basant sur l'image et le fichier de calage permet d'obtenir un signal à pas de temps constant choisi par l'utilisateur (1 heure par exemple) pour chaque marégramme. Contrairement aux données récupérées habituellement, à une heure choisie, la numérisation fournira plusieurs valeurs à savoir le minimum, la moyenne et le maximum sur le pas de temps choisi. La récupération de ces données permet de voir en fonction des marégrammes l'amplitude et parfois la période d'ondes non liées à la marée comme les tsunamis, les seiches... Ce point est très important car ces données anciennes sont parfois plus complètes que les relevés numériques actuels.
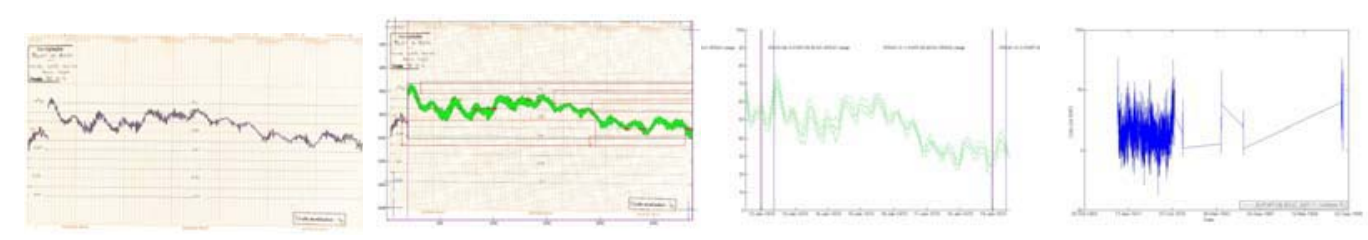

Figure 1 : Principe du logiciel NUNIEAU de gauche à droite-Image de la station de Port de Bouc appartenant au Port Autonome de Marseille, Image calée avec reconnaissance de couleur, Vérification visuelle de la continuité de la série et Compilation de toutes les données récupérées en bas à droite

Une vérification de la continuité entre les feuilles visuelles et sous forme de tableur est ensuite possible. Un retour sur le calage est parfois nécessaire en fonction du type de marégramme traité.

L'utilisation de NUNIEAU peut s'arrêter après l'étape de concaténation de l'ensemble des données numériques issues du traitement de chaque marégramme.

Quelques pistes de recherche seront évoquées pour essayer de valider à partir de codes de marée utilisés conjointement avec NUNIEAU les données numérisées.

\section{Dernières améliorations}

Les dernières améliorations apportées à NUNIEAU vont être décrites en fonction des étapes citées ci-dessus.

Un point important est la mise en ligne d'une bêta-version en Anglais. En outre, le tableau joint lors du téléchargement de l'exécutable est fourni et chacun peut ensuite modifier et retourner à l'auteur les modifications éventuelles des titres des 
différents menus du logiciel en Français, en Anglais et même créer les menus dans une autre langue. L'objectif est de partager les expériences et de normaliser les procédures de récupération de données anciennes.

\subsection{Type de données, scannage et sauvegarde des images numériques}

Les différents types (taille) de marégrammes traités sont les suivants :

- Feuille journalière, inférieure au format A4

- Feuille hebdomadaire sur 7 jours, inférieure au format A3

- Feuille hebdomadaire sur 8 jours, inférieure au format A3

- Rouleau avec des longueurs variant de $80 \mathrm{~cm}$ à plusieurs dizaines de mètres.

Ils nécessitent soit l'utilisation d'un scanner à insertion automatique (et cette étape n'est pas pénalisante en temps), soit l'utilisation d'un scanner à rouleau. L'utilisation de ce dernier est très pénalisante sur des rouleaux fins car elle nécessite parfois la découpe par pas de longueur plus court (déformation de l'image liée au scanner).

Il est possible de réaliser dès cette étape un tableau sur un tableur recensant les images scannées et indiquer certains renseignements sur l'image. NUNIEAU lit ensuite ce tableur et va modifier le nom de l'image pour la rendre compréhensible avec le nom de la station et la date au format américain (exemple : SETE-198502-03.tif). Un fichier de calage associé est aussi créé et ne nécessitera plus que deux opérations, à savoir le calage de 5 points sur la feuille et la définition d'une zone à garder. Cette étape permet une très grande industrialisation du processus sur les séries mono-signal.

\subsection{Etape de calage}

Il est utile de rappeler que les données anciennes sont souvent tachées, ont été plus ou moins bien conservées ou ont subi des écritures pour le traitement ou le stockage. Le processus se basant sur la récupération d'une plage de couleur, celleci est parfois délicate à distinguer du fond du support, d'autres écrits ou de choses encore plus courantes, comme la superposition d'enregistrements, voulue par le format (feuille 24h) ou non voulue.

Des méthodes de nettoyage manuelles sont donc à utiliser. On peut noter les éléments courant comme la sélection de zone à supprimer par cadre ou par polyligne.

D'autres éléments plus spécifiques ont été développés à savoir la sélection sur le signal à garder et la création de deux polylignes pour supprimer au-dessus et au- 
dessous.

Il est à noter que NUNIEAU peut devenir une table à digitaliser banale par récupération du signal sous la forme d'une polyligne. Cette option est la dernière à utiliser dans les cas extrêmes à savoir une incapacité du logiciel à distinguer une bande de couleur.

Une méthode de nettoyage automatique est aussi possible, à savoir que lors de la numérisation, un processus de balayage horizontal se base sur le fait que tout signal est compris entre $+/$ - trois fois son écart-type. Ce processus permet de nettoyer parfois de manière très efficace la feuille. La Figure 2 montre un exemple d'utilisation. Uniquement les données en vert sont récupérées, le données en magenta ayant été exclues automatiquement. Ce traitement a été utile pour des séries non retrouvées en couleur mais sous forme de photocopies. NUNIEAU est donc apte à gérer des données en couleur et des données en noir et blanc. Il peut aussi traiter des signaux avec une certaine épaisseur, ce qui est souvent le cas en milieu maritime malgré le filtrage des puits de tranquilisation des marégraphes (effet des ondes plus courtes que la marée).
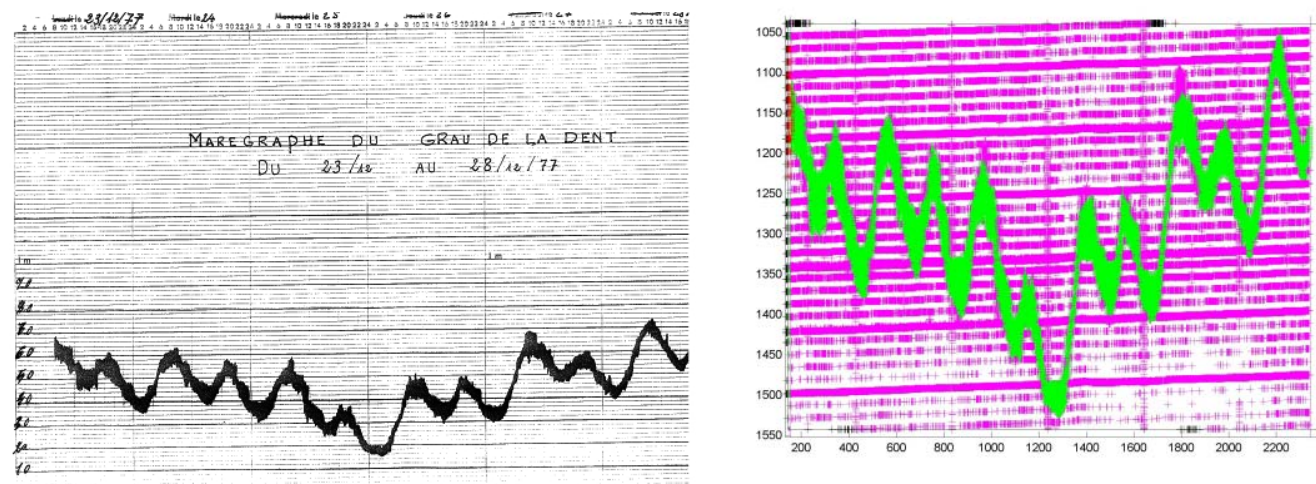

Figure 2 : exemple de traitement avec la polyligne de nettoyage automatique (Photocopies Marégramme du Grau de la Dent, Compagnie des Salins du Midi)

Sur les marégrammes multi-signaux avec des enregistrements sur 24 heures souvent utilisés en milieu à fortes marées, il est aujourd'hui intéressant d'utiliser le fait que la mesure est très proche de la marée prédite. Pour cela, il est possible d'afficher sur le marégramme le signal de la marée prédite (ou celui d'une station proche). Soit cet affichage peut permettre de nettoyer plus facilement manuellement l'image scannée, soit il est possible d'utiliser le processus de nettoyage basé sur la création de deux polylignes pour supprimer au-dessus et audessous. Cette étape qui peut se faire à la chaîne sur des marégrammes avec 5 à 10 signaux par feuille nécessite ensuite l'utilisation de la création de la polyligne 
automatique et enfin un nettoyage manuel. Dans le cas de la deuxième image de la Figure 3, l'ensemble des éléments se trouvant dans les polylignes au-dessus et endessous de la marée prédite seront supprimés.
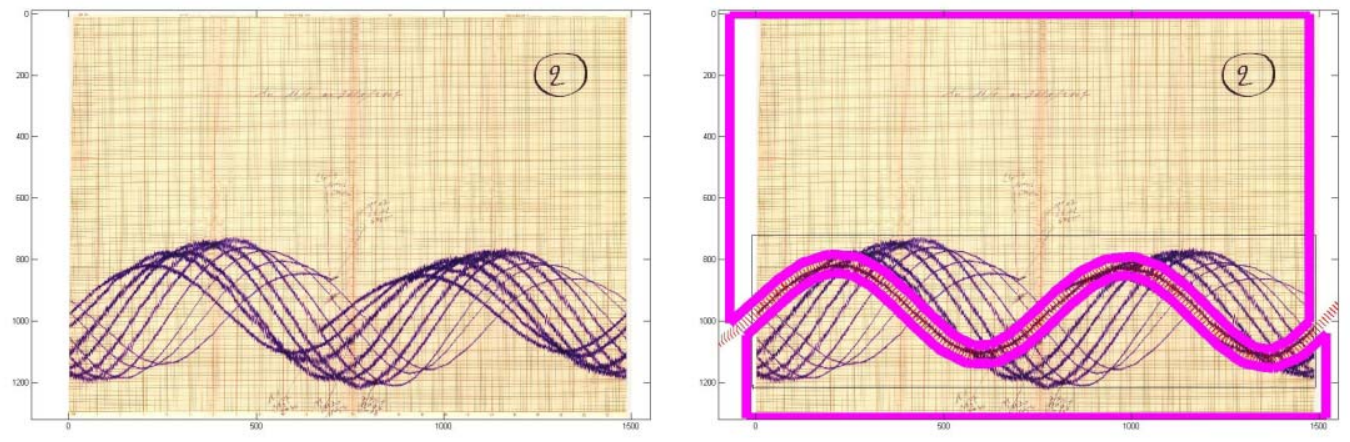

Figure 3 : Exemple d'utilisation de polyligne supprimant les éléments à une certaine distance de la marée prédite (marégramme de Port-Tudy fourni par le SHOM)

\subsection{Etape de vérification}

Elle se compose pour l'instant de deux moyens, un visuel et un sous forme de tableur.

Sous forme visuelle, cette étape permet de vérifier les possibles incohérences et a minima la continuité entre la fin d'une semaine enregistrée et le début d'une autre. Il est aussi possible de vérifier plusieurs autres séries en cours de traitement, très utile lors de la récupération de données dans des étangs par exemple avec des faibles marnages. Une autre option permet de visualiser des signaux vérifiés ou pas d'autres stations, et des traitements sur les signaux traités comme la marée ou la surcote. Ces traitements sont issus de logiciel non fournis avec NUNIEAU et mis à disposition par le SHOM (logiciel MAS) et par le LEGOS. Cette étape permet de modifier directement et de numériser à nouveau une feuille de manière instantanée pour voir la pertinence des informations apportées.

NUNIEAU permet de compiler la plupart des informations intéressantes pour qualifier des données sous la forme d'un tableau. Ce tableau peut ensuite être analysé pour voir les décalages en temps et en hauteur entre les feuilles. Un critère de qualité visuel a même été établi.

\subsection{Concaténation des données}

Cette étape est simple et permet de mettre bout à bout et dans un seul fichier les données issues de la numérisation de chaque marégramme. La dernière modification dans cette étape a été la mise en place d'une bascule temps local, 
temps universel automatique à partir des changements d'heure été/hiver intervenus depuis 1976.

\section{$4 \quad$ Synthèse des temps de travail par type de marégramme}

Il est apparu nécessaire de mettre à disposition les temps de traitement des données anciennes en fonction du format du marégramme papier. Cette synthèse des temps passés est établie à partir de l'expérience acquise au CETE Méditerranée et s'appuie sur un travail réalisé par des utilisateurs avertis et sur la base d'un nombre de feuilles (une centaine minimum) à traiter comportant des caractéristiques similaires à savoir taille, couleur du signal sur couleur de fond, échelle...

Tableau $1: 4$ Synthèse des temps de travail par type de marégramme

\begin{tabular}{|c|c|c|c|c|}
\hline $\begin{array}{c}\text { Format de } \\
\text { marégramme }\end{array}$ & $\begin{array}{l}\text { Nombre de } \\
\text { signaux }\end{array}$ & $\begin{array}{l}\text { Couleur (C) } \\
\text { ou Noir et } \\
\text { Blanc (NB) }\end{array}$ & $\begin{array}{l}\text { Temps de } \\
\text { Scannage }\end{array}$ & $\begin{array}{l}\text { Temps de } \\
\text { Numérisation }\end{array}$ \\
\hline $\begin{array}{l}24 \text { heures } \\
\quad \sim \mathrm{A} 4\end{array}$ & $\begin{array}{l}\text { Multi-signaux } \\
2 \text { à } 11 \\
\end{array}$ & $\mathrm{C}$ & \multirow{4}{*}{$\begin{array}{c}\text { Temps } \\
\text { Chargeur } \\
\text { Imprimante }\end{array}$} & $\begin{array}{c}3 \text { à } 5 \text { min par image }+ \\
\text { à } 1-5 \text { min par signal }\end{array}$ \\
\hline \multirow{3}{*}{$\begin{array}{c}7 \text { ou } 8 \text { jours } \\
\text { taille } \\
\text { inférieure } \\
\text { A3 }\end{array}$} & \multirow[b]{2}{*}{ Mono-signal } & $\mathrm{C}$ & & 3 à 10 min par image \\
\hline & & NB & & $\begin{array}{c}3 \text { à } 15 \text { min par image } \\
\text { fonction des résultats } \\
\text { de la polyligne } \\
\text { automatique }\end{array}$ \\
\hline & $\begin{array}{l}\text { Multi-signaux } \\
2 \text { à } 6\end{array}$ & $\mathrm{C}$ & & $\begin{array}{l}3 \text { à } 5 \text { min par image }+ \\
2 \text { à } 10 \text { min par signal }\end{array}$ \\
\hline \multirow[b]{2}{*}{$\begin{array}{c}\text { Rouleau } \\
\text { Taille } \\
\text { inférieure } 80 \\
\text { cm }\end{array}$} & \multirow{4}{*}{ Mono-signal } & $\mathrm{C}$ & \multirow{4}{*}{$\begin{array}{c}\text { Temps de } \\
\text { découpe et } \\
\text { de réécriture } \\
\text { des } \\
\text { informations } \\
\text { sur la feuille } \\
5 \text { min } \\
\text { /feuille a } \\
\text { minima }\end{array}$} & 3 à 10 min par image \\
\hline & & NB & & $\begin{array}{c}3 \text { à } 15 \text { min par image } \\
\text { fonction des résultats } \\
\text { de la polyligne } \\
\text { automatique }\end{array}$ \\
\hline \multirow{2}{*}{$\begin{array}{l}\text { Rouleau } \\
\text { Taille } \\
\text { supérieure } \\
80 \mathrm{~cm}\end{array}$} & & $\mathrm{C}$ & & 3 à 10 min par image \\
\hline & & NB & & $\begin{array}{l}3 \text { à } 15 \text { min par image } \\
\text { fonction de la } \\
\text { séparation automatique }\end{array}$ \\
\hline
\end{tabular}




\section{$5 \quad$ Perspectives}

Dans le cas des données récupérées autour du Golfe du Lion, il existe peu de moyens utilisés dans l'état de l'art de la marée pour qualifier les données de « fiables » ou de «non fiables » (SHOM, 2008). L'objectif du CETE et de ses maîtres d'ouvrage est de fiabiliser le plus possible les données récupérées car même si elles sont de qualité parfois médiocre, elles ont le mérite d'exister et des séries de 20 à 35 ans ne sont pas et ne seront peut-être pas si faciles à créer. Les constats issus du « Grenelle de l'Environnement » nécessitent une connaissance la plus possible, proche des territoires et actuelle. Cela pousse les techniciens et scientifiques à réfléchir à de nouvelles manières de valider les données.

Pour ce faire des démarches d'homogénéisation de données sont en cours à Météo-France sur des séries de température (CAUSSINUS et MESTRE, 2004). Nous avons la chance avec les niveaux marins de disposer d'éléments a priori « constants» par l'intermédiaire des constantes harmoniques. A partir de ce constat, nous avons essayé de voir si ces constantes harmoniques fluctuaient au cours du temps en amplitude et en phase (principalement les ondes M2, N2, S2 et K1). Nous avons mis au point une routine de calcul des constantes harmoniques par balayage en calculant sur une certaine durée et en faisant glisser cette durée d'un certain pas de temps du début à la fin de la série enregistrée. Les calculs des constantes se font à l'aide du logiciel MAS du SHOM (SHOM, 2007) et du logiciel du LEGOS.

Les résultats présentés ci-dessous sont obtenus à partir des données de PortVendres et de Sète et concernent la phase de la composante M2 de la marée. A Port-Vendres, les données proviennent des archives du SHOM (1982), de la numérisation avec NUNIEAU de 1984 à 1997 (en Temps local TL et Universel TU) du marégraphe de la DRE langeudoc-Roussillon (ex-SMNLR), du marégraphe numérique géré par le Conseil Général et du marégraphe du réseau RONIM installé depuis fin 2007. A Sète, les données proviennent de la numérisation avec NUNIEAU de 1986 à 1999 (TL et TU) du marégraphe de la DRE Languedoc-Roussillon (ex-SMNLR), d'un marégraphe numérique installé à proximité de 1991 à 2007 (avec des données de qualité médiocre dans les premières années) et du marégraphe du réseau RONIM installé depuis fin 2007.

Le balayage sur les deux séries a été effectué en calculant tous les 10 jours les valeurs des constantes sur une durée de 60 jours. Les périodes retenues sont celles avec a minima $85 \%$ de données.

Sur Figure 4, les deux séries NUNIEAU en temps local montrent une fluctuation été/hiver de l'ordre de $30^{\circ}$ de la phase de M2. Une fluctuation de la phase de M2 de $28.98410422^{\circ}$ correspond à un décalage d'une heure réelle dans la série. Le passage en Temps Universel stabilise la phase de la constante de la marée M2 et 
devient comparable à celle calculée à partir des marégraphes RONIM (2 à 3 points calculés uniquement) ou du milieu de la période du marégraphe DRE LR numérique de Sète. Il apparaît donc que le changement établi sans aucune justification préalable (note, suivi du marégraphe) est bien réel et justifié par cette visualisation. Il existe cependant plus de fluctuations que dans les données numériques de Sète et on peut noter des fluctuations inexpliquées plus importantes de la phase de M2 sur la série de Port-Vendres entre 1987 et 1989. Celles-ci correspondent à des dérives de l'horloge du marégraphe et problématique pour les calculs des surcotes ou décotes météorologiques.
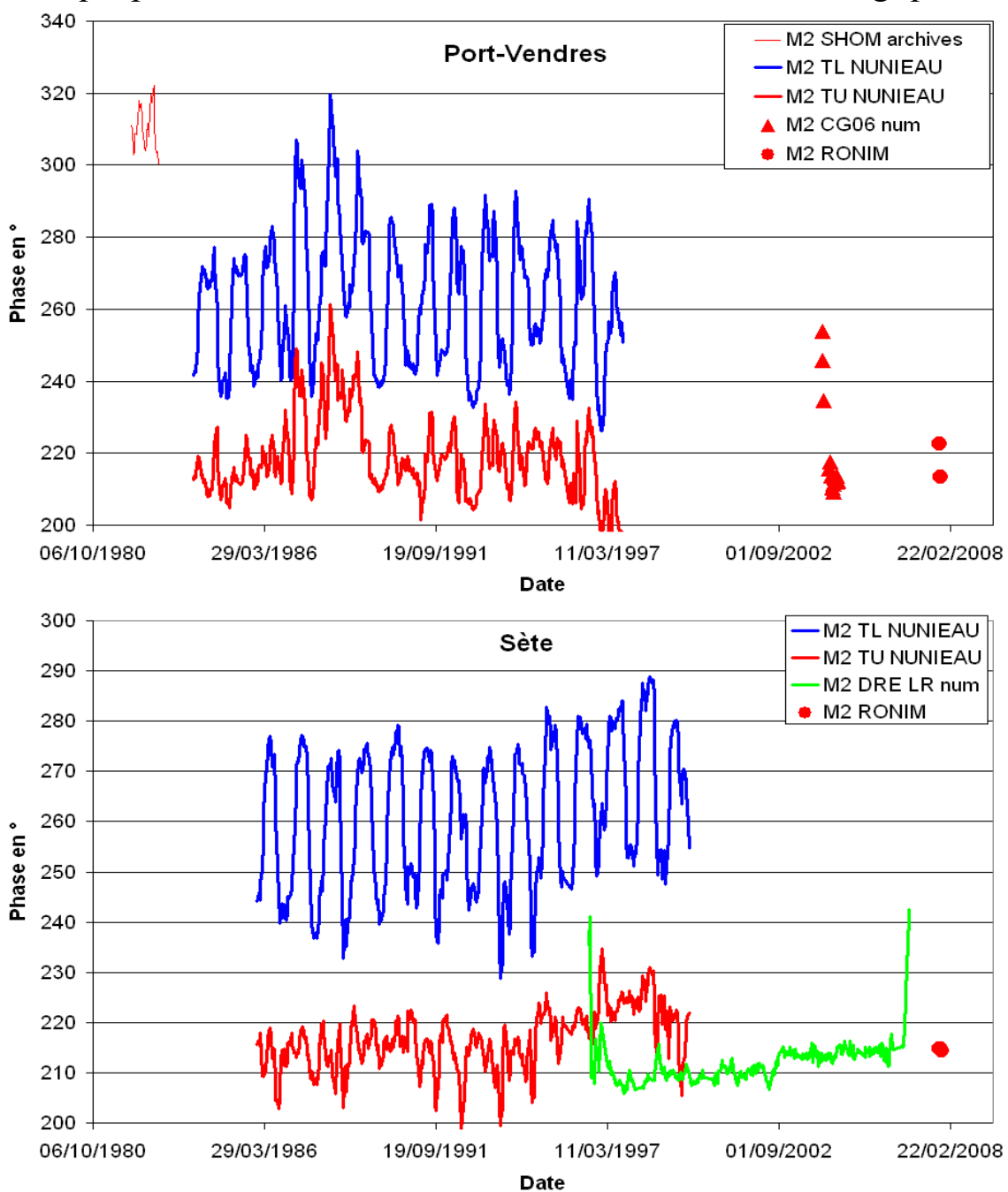

Figure 4 : Phase de M2 sur les données de Port-Vendres et de Sète

D’autres types d'analyses sur la marée vont être menés, les incohérences ou incertitudes visuelles et statistiques seront intégrées dans les fichiers de calage de NUNIEAU (décalage en temps, en altitude, commentaires). L'objectif est la traçabilité complète du processus de récupération des données. 


\section{$6 \quad$ Conclusions}

Cet article a permis de montrer la pertinence d'un outil et surtout d'une méthode moderne de récupération de données anciennes. Cet outil utilisé ici dans le cadre de la marée est utilisable pour des données de rivières ou des séries de température. Ses dernières améliorations et la synthèse des temps prévus par type de marégrammes permettent de définir des possibilités de récupération de données à la fois pour des Universitaires et pour des donneurs d'ordre ou des bureaux d'études. La traduction possible dans diverses langues en fait un outil ouvert à une plus large communauté.

Il est utile de rappeler quelques chiffres de données archivées sous forme papier en France dans quelques services connus du CETE Méditerranée : plus de 150 années au Port Autonome de Marseille, et près de 400 ans à la Direction Régionale de l'Environnement d'Ile de France et dans les mêmes ordres de grandeur pour le Service de Prévision de Crues Med-Ouest.

\section{$7 \quad$ Remerciements}

L'auteur tient à remercier les divers maîtres d'ouvrages à savoir la DRE Languedoc-Roussillon, le SCHAPI et de la DDE des Bouches du Rhône, tous organismes du Ministère de l'Ecologie, de l'Energie, du Développement durable et de l'Aménagement du territoire.

Le SHOM, le LEGOS et SONEL sont également remerciés pour leurs échanges scientifiques et techniques avec le CETE Méditerranée.

\section{$8 \quad$ Références bibliographiques}

1 CAUSSINUS H. MESTRE O. (2004), Detection and correction of artificial shifts in climate, Appl. Statist.53, 3: 405-425

2 PONS F., (2008), Utilisation des données anciennes pour la connaissance des risques de submersions marines sur le Golfe du Lion, Colloque SHF - Nouvelles approches sur les risques côtiers - Paris 30-31 Janvier 2008

3 POUVREAU N., (2008), Chazallon, père du premier réseau marégraphique en France, Lettre du Réseau RONIM, Mars 2008 p2-3, UMR CNRS 6250 LIENSS, Université de La Rochelle

4 SHOM/DO/MIP (2007), Mode Opératoire - Documentation de Mas, État : Rédaction Version 1.0, 177p

5 SHOM/DO/MIP (2008), Procédure Spécifique - Traitement des mesures de marée par la division MIP, État : Rédaction Version 2, 25p

6 ULLMANN A., PONS F., MORON V. (2005), Tool Kit Helps Digitize Tide Gauge Records, Eos Trans. AGU, 86(38), doi:10.1029/2005EO380004. 